\title{
miR-455 inhibits cell proliferation and migration via negative regulation of EGFR in human gastric cancer
}

\author{
TAO NING ${ }^{*}$, ZHIJUAN PENG ${ }^{*}$, SHUANG LI*, YANJUN QU*, HAIYANG ZHANG*, \\ JINGJING DUAN, XINYI WANG, HAIOU YANG, RUI LIU, TING DENG, MING BAI, \\ YI WANG, YIRAN SI, LE ZHANG, XIA WANG, SHAOHUA GE, \\ LIKUN ZHOU, GUOGUANG YING and YI BA
}

Tianjin Medical University Cancer Institute and Hospital, National Clinical Research Center for Cancer, Key Laboratory of Cancer Prevention and Therapy, Tianjin, Tianjin's Clinical

Research Center for Cancer, Tianjin 300060, P.R. China

Received October 27, 2016; Accepted November 24, 2016

DOI: $10.3892 /$ or.2017.5657

\begin{abstract}
Epidermal growth factor receptor (EGFR) plays an important role in various types of cancer. However, the therapeutic agents that target EGFR have not produced favorable results in gastric cancer. miRNAs are known to regulate gene expression at the post-transcriptional level. We wondered if miRNAs could be potential therapeutic agents for the targeted therapy against EGFR. In this study, we found an increase in the copies of EGFR mRNA and the upregulated expression of the EGFR protein in gastric cancer tissues compared with normal gastric tissues. Both bioinformatic analysis and luciferase reporter assay revealed that miR- 455 could directly bind with the 3'-untranslated region (3'UTR) of EGFR mRNA. Subsequently, in vitro studies were conducted to identify the effects of miR-455 on cell proliferation and migration and further confirm the cancer-promoting role of EGFR in gastric cancer. The results revealed that miR- 455 negatively regulated EGFR expression at the post-transcriptional level, thus suppressing cell growth and migration. To conclude, our results offer a potential targeted therapeutic method against EGFR in gastric cancer mediated by miR- 455 .
\end{abstract}

Correspondence to: Professor Yi Ba or Professor Guoguang Ying, Tianjin Medical University Cancer Institute and Hospital, National Clinical Research Center for Cancer, Key Laboratory of Cancer Prevention and Therapy, Tianjin, Tianjin's Clinical Research Center for Cancer, 18 Huan Hu Xi Road, Tianjin 300060, P.R. China

E-mail: bayi@tjmuch.com

E-mail: yingguoguang163@163.com

"Contributed equally

Abbreviations: EGFR, epidermal growth factor receptor; GAPDH, glyceraldehyde-3-phosphate dehydrogenase; 3'UTR, 3'-untranslated region

Key words: gastric cancer, EGFR, miR-455, cell proliferation, migration

\section{Introduction}

Gastric cancer is one of the most common malignancies worldwide and causes one million cancer-related deaths each year (1). Advanced disease stage and metastasis are often present at diagnosis due to the lack of specific symptoms and limitation of early diagnostic methods, resulting in a poor prognosis with a less than $10 \% 5$-year survival rate (2). As an effective means of treatment for gastric cancer, molecular-targeted therapy is important both in clinical research and practice.

Epidermal growth factor receptor (EGFR) is a crucial member of the HER/erbB family of receptor tyrosine kinases (RTKs), which includes HER1 (EGFR/erbB1), HER2 (neu, erbB2), HER3 (erbB3) and HER4 (erbB4) (3). The EGFR signaling pathway is initiated by the binding of EGF, one of the major ligands, to EGFR, leading to the activation of downstream signaling cascades, and finally to the proliferation, invasion and metastasis of tumor cells, the inhibition of apoptosis of tumor cells, and the promotion of angiogenesis of tumors, making it a paramount target in various types of cancer (4-6). Studies have revealed a high level of expression of EGFR in lung (7), breast (8), gastric (9), prostate (10) and head and neck cancer (11), and a high expression indicates a worse prognosis (12). There are two types of anti-EGFR agents designed to inhibit the activity of EGFR: small-molecule tyrosine kinase inhibitors (TKIs) and monoclonal antibodies. But, unlike their fruitful efficacy in lung cancer, neither type of agent has brought patients with gastric cancer survival benefits according to the present clinical research (13). Thus, further research in anti-EGFR targeted therapy in gastric cancer is warranted.

MicroRNAs have been known to play important roles in the tumorigenesis and progression of various types of cancer. Studies have shown that aberrant expression is one of the mechanisms of carcinogenesis, invasion and metastasis of gastric cancer $(14,15)$. Therefore, further investigation of miRNAs in gastric cancer could help us to better understand the initiation and the development of gastric cancer and may provide new approaches in targeted therapy. Dysregulation of EGFR and miRNAs has been known to be interrelated with 
the carcinogenesis and development of gastric cancer. But few studies have revealed the relationship between miRNAs and EGFR in gastric cancer $(16,17)$.

In this study, bioinformatic prediction and experimental evidence were provided for the specific binding between miR-455 and EGFR. Then the suppression of EGFR protein expression by miR- 455 was evaluated. The tumor-promoting effect of EGFR was further assessed in gastric cancer cells. Our findings provide evidence of the function of miR-455 in gastric cancer and help to better understand the modulation of signaling pathways in gastric cancer.

\section{Materials and methods}

Human tissues. Histologically confirmed gastric cancer tissues and paired adjacent non-cancerous tissues were obtained from patients who underwent partial or total gastrectomy at Tianjin Medical University Cancer Institute and Hospital (Tianjin, China). Each cancer tissue was confirmed as adenocarcinoma pathologically. Written informed consent was provided by each patient, and all aspects of this study were approved by the Ethics Committee of Tianjin Medical University Cancer Institute and Hospital. Tissues were immediately frozen in liquid nitrogen after surgery and were stored at $-80^{\circ} \mathrm{C}$.

Cell line and culture. The human gastric cancer cell line SGC-7901 and human embryo kidney epithelial cell line HEK293T were resuscitated from the Cancer Cell Biological Laboratory of Tianjin Medical University Cancer Institute and Hospital. SGC-7901 cells were cultured in RPMI-1640 medium (Gibco, Rockville, MD, USA) and HEK-293T cells were cultured in Dulbecco's modified Eagle's medium (DMEM) (Gibco), both containing 10\% fetal bovine serum (FBS) (Gibco) and $1 \%$ penicillin/streptomycin (Solarbio Science \& Technology Co., Ltd., Beijing, China) in a humidified incubator at $37^{\circ} \mathrm{C}$ with $5 \% \mathrm{CO}_{2}$. Cells were grown in sterilized culture dishes.

Cell transfection. Cell transfection was conducted through Lipofectamine 2000 (Invitrogen, Carlsbad, CA, USA) method after the cells were seeded in appropriate plates. miR-455 mimics and miR-455 inhibitor were purchased with the corresponding controls (RiboBio Co., Ltd., Guangzhou, China) and were used to upregulate or downregulate the level of miR-455. The overexpressing lentivirus and the control lentivirus were purchased from GenePhama (Shanghai, China) and were used to upregulate or downregulate the expression of EGFR. Both the overexpression and control lentivirus contained green fluorescence for the identification of the infection efficiency after the cells were infected for 36-72 $\mathrm{h}$. The siRNA sequence targeting human EGFR and a control siRNA were purchased from Santa Cruz Biotechnology (sc-29301). The cells were harvested after transfection or infection to isolate total RNA and total cell lysates for qPCR and western blot analysis, respectively.

RNA isolation and quantitative RT-PCR. TRIzol reagent (Invitrogen) was used to isolate the total RNA of the cultured SGC-7901 cells and the obtained tissues according to the manufacturer's protocol. Nanodrop 1000 spectrophotometer
(Thermo Fisher Scientific, USA) was used to confirm the quality and the concentration of the isolated RNA.

First-strand cDNA was synthesized from $1 \mu \mathrm{g}$ of total RNA through reverse transcription reaction. The reaction was carried out as follows: $16^{\circ} \mathrm{C}$ for $15 \mathrm{~min}, 42^{\circ} \mathrm{C}$ for $60 \mathrm{~min}, 85^{\circ} \mathrm{C}$ for $5 \mathrm{~min}$ and maintained at $4^{\circ} \mathrm{C}$. The cDNA was stored at $-20^{\circ} \mathrm{C}$ after the reaction.

The level of miR-455 was detected by TaqMan miRNA probes (Applied Biosystems, USA). Gene-specific PCR products were assayed using a CFX96 real-time PCR system. The reaction conditions were initiated by a 5 -min hold at $95^{\circ} \mathrm{C}$, followed by 40 cycles of denaturation at $95^{\circ} \mathrm{C}$ for $15 \mathrm{sec}$ and annealing/extension at $60^{\circ} \mathrm{C}$ for $1 \mathrm{~min}$. All the reactions were conducted three times. U6 snRNA was used as an internal control for miRNA. The mRNA levels of EGFR were analyzed by SYBR-Green fluorescent method and the final results were normalized to glyceraldehyde-3-phosphate dehydrogenase (GAPDH). The reaction was as follows: $95^{\circ} \mathrm{C}$ for $30 \mathrm{sec}$ following 40 cycles of $95^{\circ} \mathrm{C}$ for $5 \mathrm{sec}$ and $60^{\circ} \mathrm{C}$ for $30 \mathrm{sec}$. The relative levels of the miRNA and mRNA were calculated by $\Delta \mathrm{Ct}$ (cycle threshold) and normalized to the control using the equation $2^{-\Delta \mathrm{Ct}}, \Delta \mathrm{Ct}=\mathrm{Ct}_{\text {gene }}-\mathrm{Ct}_{\text {control }}$.

Protein extraction and western blot analysis. Lysates were obtained after the cells and tissues were lysed in RIPA buffer with freshly added PMSF. Subsequently, $\sim 50 \mu \mathrm{g}$ of protein was separated using SDS-PAGE gels and transferred to PVDF membranes. Immunoblotting was then conducted at $4^{\circ} \mathrm{C}$ overnight with monoclonal anti-EGFR antibodies $(1: 100,000$; Abcam) and monoclonal anti-GAPDH antibodies (1:2,000; Santa Cruz Biotechnology) after blocking with $2 \%$ BSA. An enhanced chemiluminescence system kit (Millipore, USA) was used to visualize the membranes after incubation with HRP-coupled anti-mouse/rabbit IgG (1:2,000; Santa Cruz Biotechnology, USA) at $37^{\circ} \mathrm{C}$ for $1 \mathrm{~h}$ with the secondary antibody.

Luciferase reporter assay. The amplified PCR products of human wild-type EGFR and a mutant EGFR in which the predicted 3'-untranslated region (3'UTR) miR-455 targeting regions were inserted into the reporter plasmid (Ambion, USA). Lipofectamine 2000 (Invitrogen) method was used for cell transfection with a reporter plasmid, $\beta$-galactosidase expression vector (Ambion), miR-455 mimics, miR-455 inhibitors, or a corresponding negative control RNA.

Cell proliferation assay. EdU (RiboBio) was added to the cell culture medium at a final concentration of $50 \mu \mathrm{M}$ for a $5-\mathrm{h}$ incubation at $37^{\circ} \mathrm{C}$ after transfection or infection. After fixation in $4 \%$ paraformaldehyde and treatment with $0.5 \%$ Triton- $X$ for $15 \mathrm{~min}$, the cells were incubated at room temperature in the dark with Apollo after treatment with $4 \%$ paraformaldehyde for fixation and $0.5 \%$ Triton-X for permeabilization. Nuclei were stained by Hoechst. Five random fields were selected to calculate the ratio between EdU-labeled cells and the total cells.

Cell migration assay. Cells $\left(10^{5}\right)$ were seeded on a Boyden chamber with $200 \mu 1$ of serum-free medium after transfection and infection. Medium $(600 \mu \mathrm{l})$ with $10 \%$ serum was 

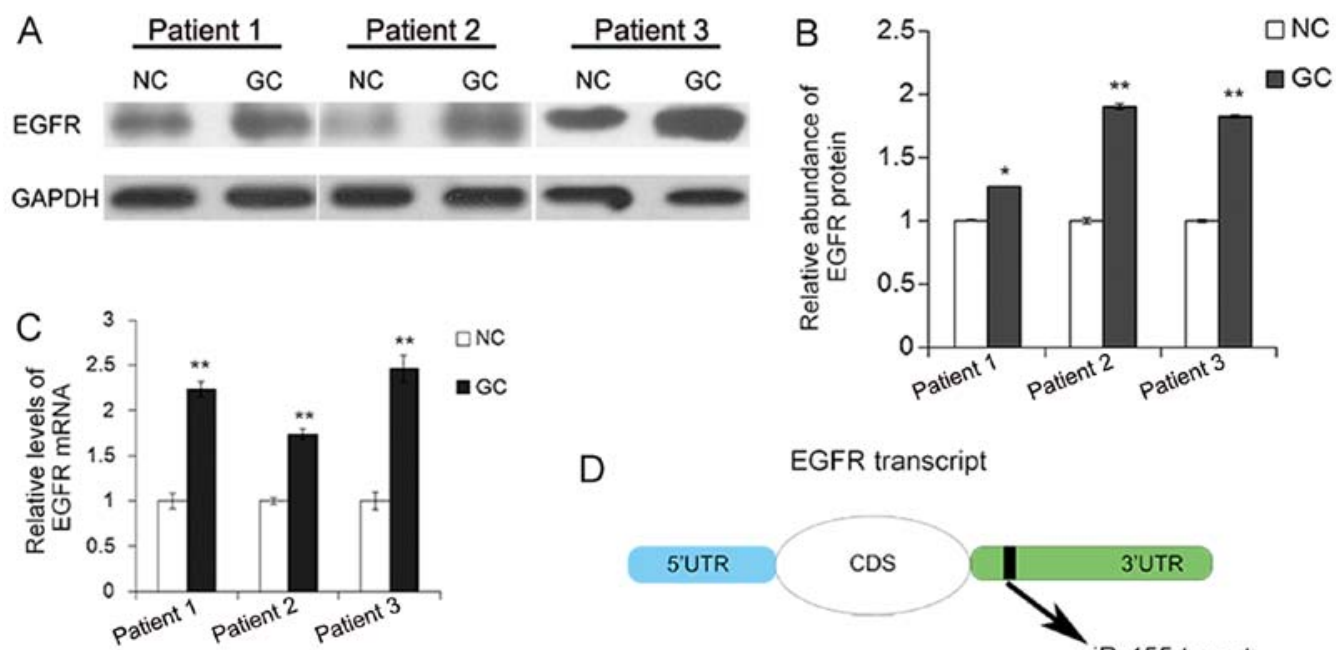

$\mathrm{D}$

EGFR transcript

E
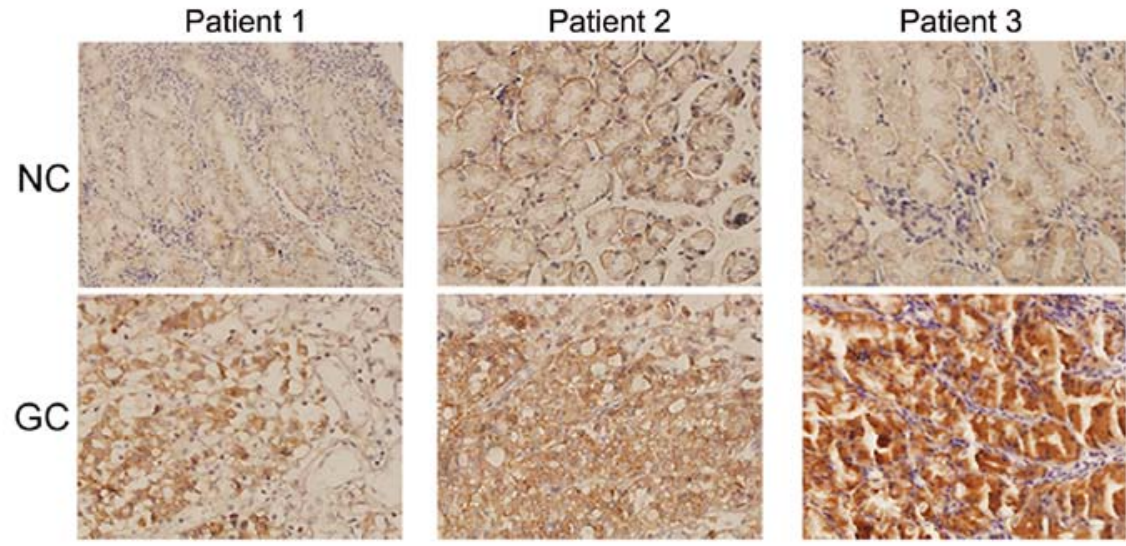

Figure 1. Inverse correlation between epidermal growth factor receptor (EGFR) and miR-455 in human gastric cancer (GC) tissues. (A) Western blot analysis revealed the expression of EGFR in GC tissues and the paired non-cancerous tissues ( $n=3$ ). (B) Quantitative analysis of A. (C) Relative EGFR mRNA levels in GC tissues (n=3). (D) The predicted binding sites of miR-455 in the mRNA of EGFR. (E) Immunohistochemistry of the paraffin-embedded human gastric cancer tissues and paired non-cancerous tissues $(n=3)$. $N C$ is the paired non-cancerous group of GC. ${ }^{* *} p<0.01$.

added to the lower chamber for chemotaxis. The membranes of the Boyden chambers were fixed and stained after $24 \mathrm{~h}$ of incubation. Five random visual fields were selected for analysis.

Wound scratch assay. SGC-7901 cells that had undergone different treatments were seeded in 6-well plates. Each well was scraped with a $20 \mu 1$ pipette tip to create 1 or 2 linear regions devoid of cells after the cells reached $90 \%$ confluence. RPMI-1640 medium with 2\% FBS (both from Gibco) were used subsequently for cell culture. We monitored wound healing at 0,12 , and $24 \mathrm{~h}$ after scraping. Five random fields of each well were selected for analysis.

Immunohistochemical assay. Paraffin-embedded specimens of gastric cancer tissues and the paired non-cancerous tissues were incubated with an anti-EGFR monoclonal antibody (1:100; Abcam) following antigen retrieval. The DAB system (Zhongshan Jinqiao, China) was used to identify the positive reaction. Five random fields were selected for each specimen.

Bioinformatics prediction of miRNA target. TargetScan (http://www.targetscan.org/), PicTar (http://pictar.mdc-berlin. de/) and miRanda (http://www.microrna.org/) were combined for the prediction of the target of miR-455.

Statistical analysis. Each experiment was performed at least three times. $\mathrm{P}<0.05$ was considered as statistically significant and differences were assessed using the Student's t-test. Data are expressed as the median values \pm SE and analyzed using the Student's t-test. In this study, ' $*$ ' indicates $\mathrm{P}<0.05$, ' $* *$ ' indicates $\mathrm{P}<0.01$, and ' $* * *$ ' indicates $\mathrm{P}<0.001$.

\section{Results}

EGFR protein levels are upregulated in gastric cancer tissues. The expression pattern of EGFR in human gastric cancer tissues was first evaluated by western blot analysis. As previously reported, the EGFR protein level was significantly upregulated in the gastric cancer tissues (Fig. 1A and B). As is well known, an increase in EGFR copies is one of the causes for the upregulation of EGFR. Here, the mRNA level was upregulated in the cancerous tissues (Fig. 1C), which may be due to a variety of factors. The predicted target of miR-455 on the transcript of EGFR is shown in Fig. 1D. IHC assays revealed that EGFR distributed in the cytoplasm and cancer 
A

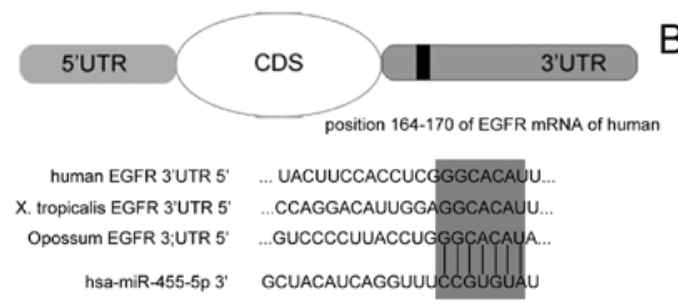

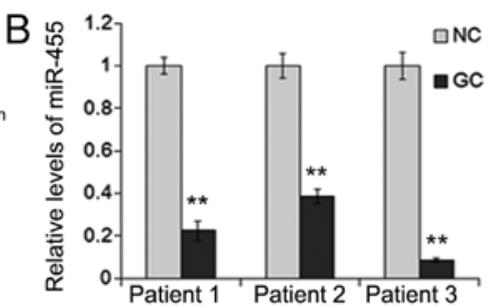

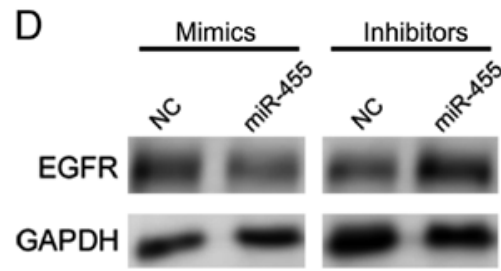

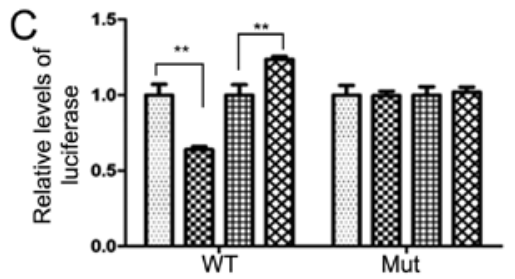
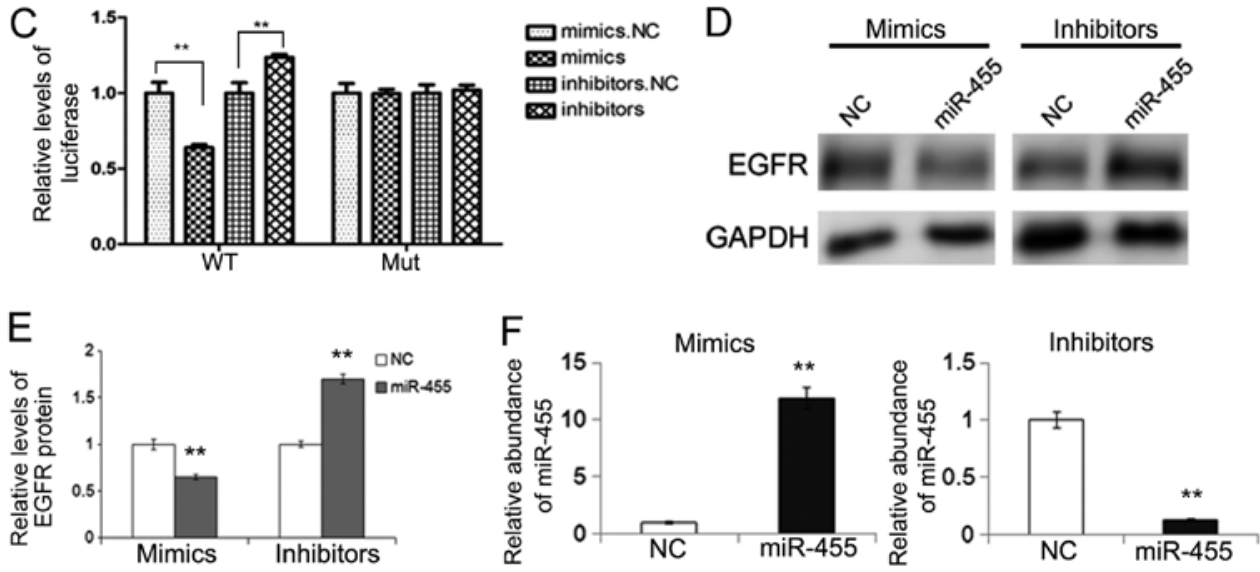

Figure 2. miR-455 regulates epidermal growth factor receptor (EGFR) expression in gastric cancer (GC) cells. (A) Schematic description of the base-pairing interaction between miR-455 and EGFR mRNA. (B) Relative levels of miR-455 in GC tissues and para-carcinoma tissues (n=3). (C) Direct recognition of EGFR by miR-455. HEK293T cells were co-transfected with firefly luciferase reporters containing either wild-type (WT) or mutant (Mut) EGFR 3'UTR with miR-455 mimics and inhibitors. An interaction between miR-455 and the target was evident ( $\mathrm{n}=3$ ). (D) The suppression of EGFR expression by miR-455 in SGC7901 cells ( $n=3)$. (E) Quantitative analysis of D (n=3). (F) Quantitative RT-PCR analysis of the relative miR-455 levels in SGC-7901 cells transfected with miR-455 mimics $(n=3)$ and inhibitors $(n=3)$. NC is the corresponding negative control of mimics or inhibitors. ${ }^{* *} \mathrm{p}<0.01$.

tissue specimens exhibited a higher positive rate, compared with paired non-cancerous tissues, which was consistent with previous studies (Fig. 1E).

miR-455 acts as a potential upstream regulator of EGFR. Bioinformatic prediction indicated a direct binding of miR-455 to the region in the 3'UTR of EGFR mRNA. The binding sites are conserved among several species (Fig. 2A). The evaluation of miRNA levels using real-time PCR revealed that compared with para-carcinoma tissues, miR-455 was markedly downregulated in the gastric cancer tissues (Fig. 2B). The inverse expression patterns of the EGFR protein and miR-455 prompted further investigation into the potential effect of miR-455 on EGFR. Therefore, miR-455 was chosen for further experiments to identify its role in gastric cancer.

Validation of EGFR as a direct target of miR-455. Direct evidence of the combination of miR-455 and EGFR was needed despite the bioinformatic prediction and the inverse correlation between miR-455 and the EGFR protein level. A dual luciferase reporter assay was conducted to evaluate the direct interaction between miR-455 and EGFR. The relative luciferase activity was clearly suppressed by the co-transfection of miR-455 mimics and the luciferase reporters containing the predicted binding region of the wild-type 3'UTR of EGFR. However, the suppressive effect was lost when the binding site was mutated. Furthermore, the co-transfection of miR-455 inhibitors and the reporter plasmid with the wild-type EGFR 3'UTR resulted in an increase in luciferase activity (Fig. 2C).
The expression of EGFR protein and mRNA were evaluated when SGC-7901 cells were transfected with miR-455 mimics or inhibitors, which was confirmed by qRT-PCR (Fig. 2F). As shown in Fig. 2D and E, upregulation of miR-455 led to a sharp decrease in the EGFR protein, whereas downregulation of miR-455 enhanced the expression of EGFR in the gastric cancer cells.

The results indicated that miR-455 regulated EGFR by directly binding with the specific region of the 3'UTR of EGFR mRNA, thus rendering it as an important regulator of EGFR.

Upregulation of miR-455 inhibits the proliferation and migration of SGC-7901 cells. To further assess the role of miR-455 in gastric cancer cells, EdU cell proliferation assay, Transwell migration assay and wound healing assay were performed respectively.

The cell proliferation rate via the Cell-Light EdU DNA cell kit was used to identify the proliferation ability of SGC-7901 cells after treatment with miR-455 mimics or inhibitors. As shown in Fig. 3B and D, upregulation of miR-455 resulted in a sharp decrease in cell proliferation, whereas downregulation of miR-455 led to an increase in cell proliferation.

The effects of miR-455 on cell migration were evaluated through Transwell assays. As was expected, upregulation of miR-455 expression strongly inhibited the migration of gastric cancer cells, whereas the downregulation of miR-455 expression promoted cell migration (Fig. 3A and C).

A wound-healing assay was also conducted to further examine the migration ability of SGC-7901 cells. Similar to the 

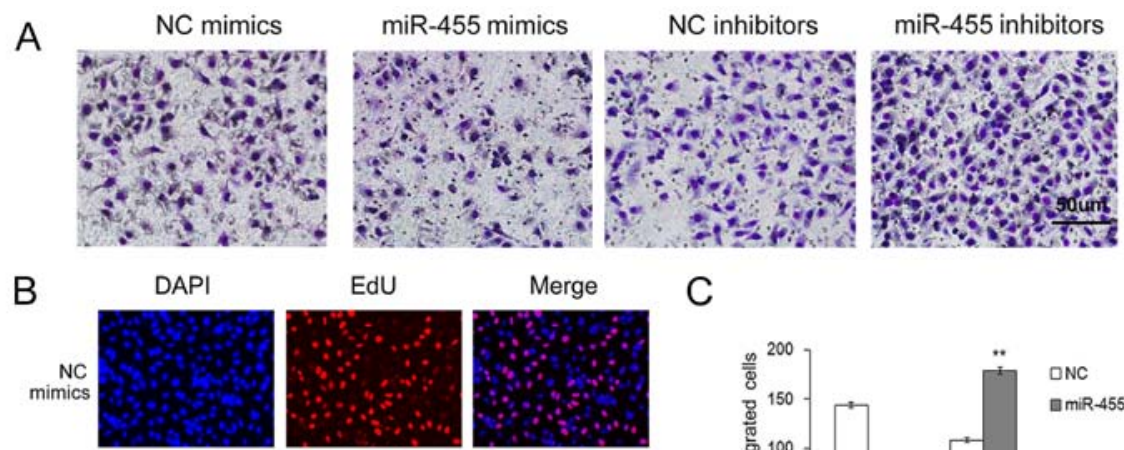

EdU

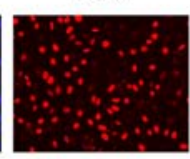

Merge

C
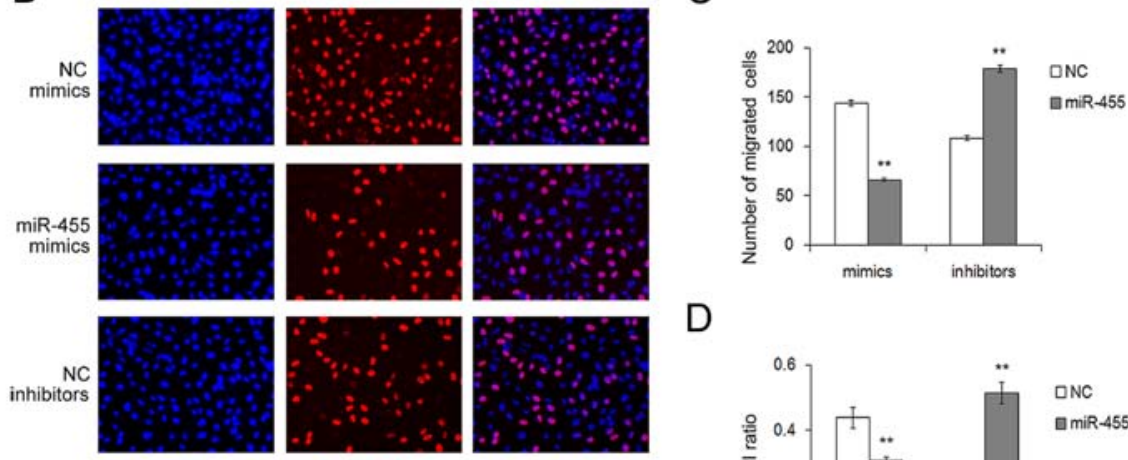

D
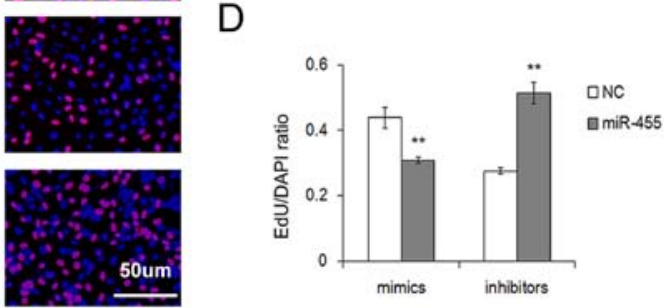

$\mathrm{E}$

NC mimics

miR-455 mimics

NC inhibitors

miR-455 inhibitors

$\mathrm{Oh}$
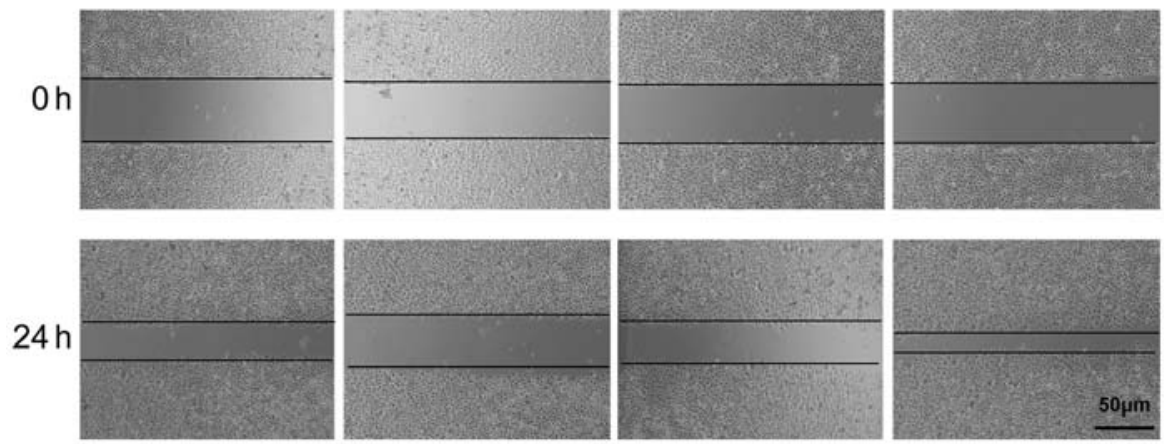

Figure 3. miR-455 regulates the proliferation and migration of gastric cancer (GC) cells. (A) Transwell assays demonstrated that miR-455 promotes the migration of GC cells. Overexpression of miR-455 promotes cell migration, whereas low levels of miR-455 suppress GC cell migration in GC ( $\mathrm{n}=3$ ). (C) Quantitative analysis of $\mathrm{A}(\mathrm{n}=3)$. (B) EdU assays demonstrated that miR-455 promotes the proliferation of GC cells. Overexpression of miR-455 promotes cell proliferation, whereas low levels of miR-455 suppress GC cell proliferation ( $\mathrm{n}=3$ ). (D) Quantification of C ( $\mathrm{n}=3$ ). (E) Validation of miR-455-mediated cell migration by the wound-healing method $(\mathrm{n}=3)$. NC is the corresponding negative control of mimics or inhibitors. ${ }^{* *} \mathrm{p}<0.01$.

A
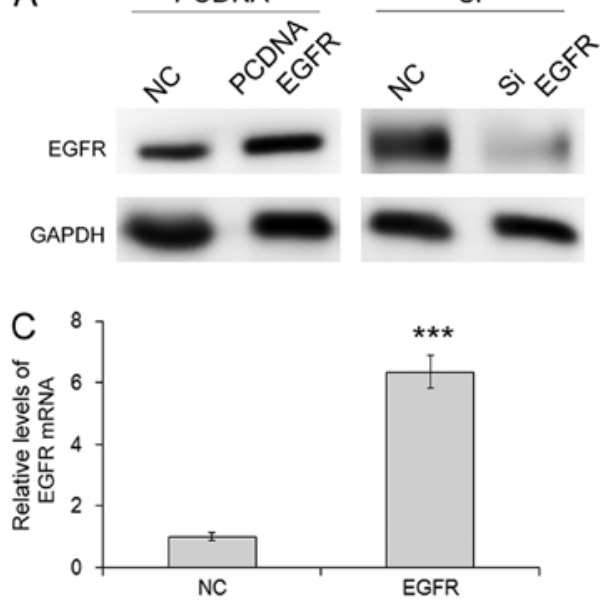

B
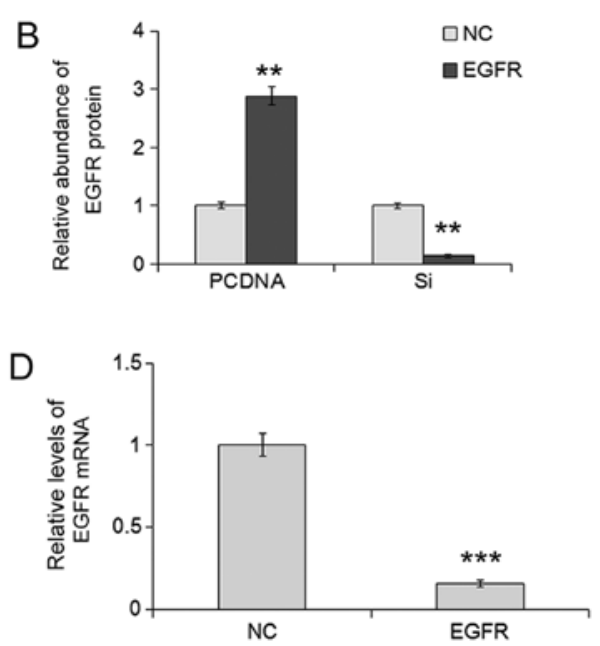

Figure 4. Knockdown of epidermal growth factor receptor (EGFR) in SGC7901 cells. (A and B) Western blot analysis of EGFR expression in EGFRoverexpressing or -silenced cells by overexpression lentivirus or siRNA. SGC-7901 cells were transfected with plasmid or siRNA, and the protein levels were assessed $(n=3)$. (C and D) The mRNA levels of A were quantified $(n=3)$. Si NC is the negative control of the siRNA of EGFR. PCDNA NC is the negative control of the EGFR overexpression lentivirus. ${ }^{* *} \mathrm{p}<0.01$. 


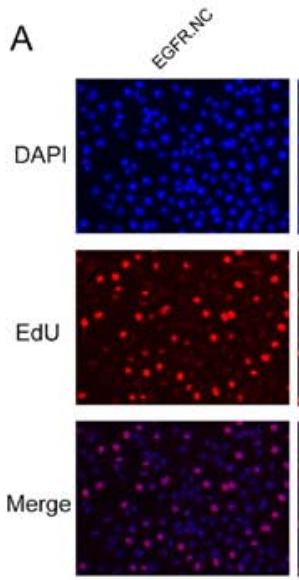

C

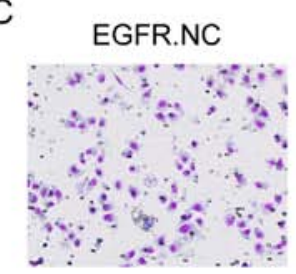

Si.NC

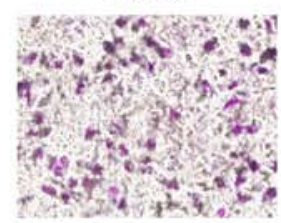

E
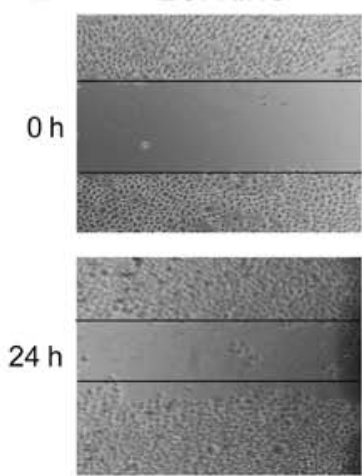
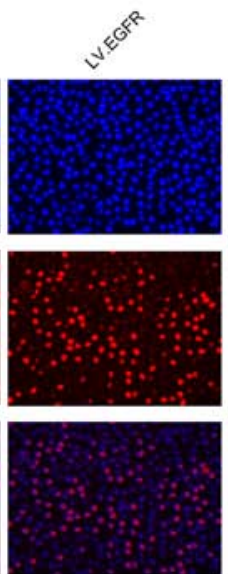

LV.EGFR

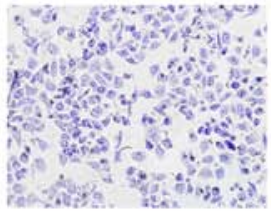

Si.EGFR

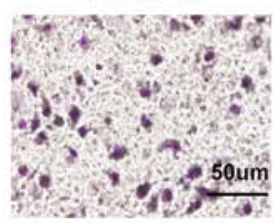

LV.EGFR
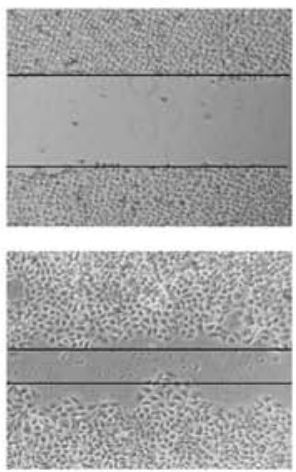

$5^{5}$
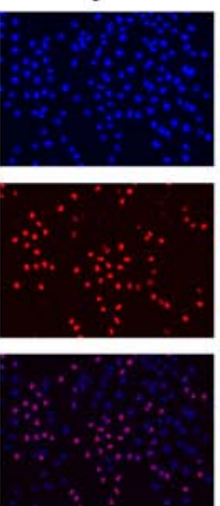

D
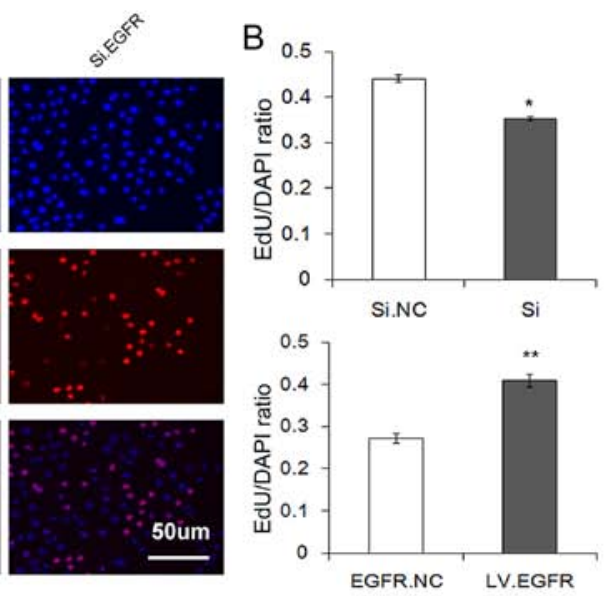

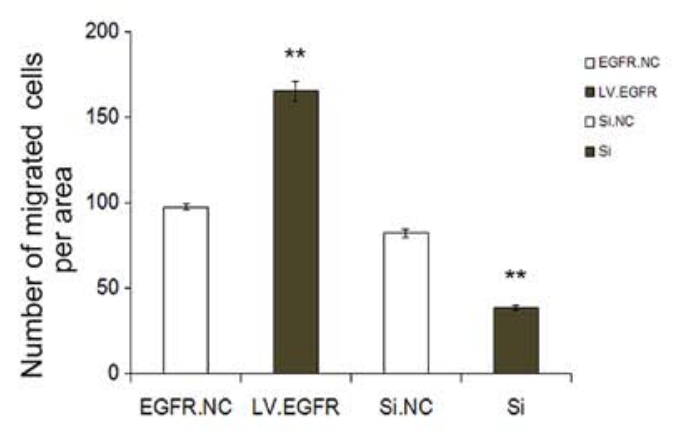

Si.NC

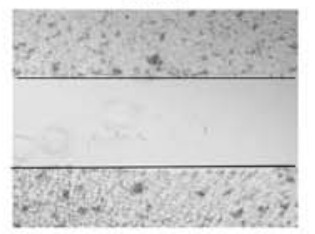

Si.EGFR
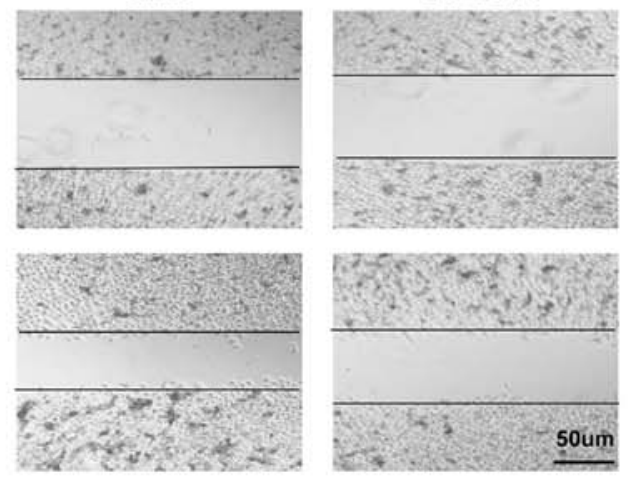

Figure 5. Overexpression or silencing of epidermal growth factor receptor (EGFR) regulates cell proliferation and migration in SGC-7901 cells. (A) Silencing of EGFR clearly promoted the proliferation of SGC7901 cells, whereas overexpression of EGFR inhibited cell proliferation ( $\mathrm{n}=3$ ). (B) Quantitative analysis of A $(n=3)$. (C) Transwell assays demonstrated that knockdown of EGFR strongly enhanced cell migration in GC cells, whereas overexpression of EGFR inhibited cell migration ( $n=3$ ). (D) Quantitative analysis of C. (E) Validation of cell migration by wound-healing method after downregulation or upregulation of the expression of EGFR ( $n=3)$. Si.NC is the negative control of the siRNA of EGFR. EGFR.NC is the negative control of the EGFR overexpression lentivirus. $* * \mathrm{p}<0.01$.

Transwell assay result, upregulation of miR-455 significantly suppressed the migration of gastric cancer cells (Fig. 3E).

These aforementioned in vitro results indicated that miR-455 exerts a cancer-suppressive role in gastric cancer cells and provides evidence that miRNA participates in the processes of gastric cancer.

Overexpression of EGFR promotes proliferation and migration of SGC-7901 cells. Next, we investigated the effects of EGFR on cell proliferation and migration by overexpressing and silencing EGFR. An overexpression lentivirus was constructed to overexpress EGFR, and the siRNA sequence targeting human EGFR was designed to knock down the expression of EGFR. It was revealed in our study that infection of the EGFR overexpression lentivirus led to a significant increase or siRNA led to a decrease in protein and mRNA levels in SGC-7901 cells, compared with the control (Fig.4). Knockdown of EGFR by siRNA downregulated the protein expression and suppressed the proliferation and migration, whereas overexpression of EGFR resulted in a higher rate of proliferation and migration. In addition, we used EdU assay, Transwell assay and woundhealing assay to assess SGC-7901 cells in which EGFR was knocked down and the cells exhibited a significantly lower rate of proliferation and decreased migration, whereas the cells 
overexpressing EGFR exhibited a significantly higher rate of proliferation and decreased migration (Fig. 5).

\section{Discussion}

A substantial number of studies on certain miRNAs or miRNA patterns have been published in recent years, indicating that miRNAs play an important role in the initiation and development of gastric cancer. For example, miR-1, miR-206, miR-34a and miR-144 directly target the MET gene and downregulate its expression, thus inhibiting gastric cancer cell proliferation and migration (18-21). miR-196a/-196b promotes cell metastasis by targeting radixin in gastric cancer (22). miRNAs are also relevant to the chemosensitivity of cancer cells. For example, miR-23b-3p inhibited autophagy by targeting ATG12 and HMGB2 and made gastric cancer cells sensitive to chemotherapy (23). miR-135 can reverse resistance to chemotherapy and promote apoptosis by negative regulation of MCL1 (24). Therefore, identifying the function of miRNA can help us to better understand the carcinogenesis and progression of gastric cancer.

In the present study, bioinformatic prediction for upstream miRNAs of EGFR and an inverse expression pattern of predicted miR-455 and EGFR in human gastric cancer and para-carcinoma tissues indicated a potential binding of miR-455 with the 3'UTR of EGFR mRNA. Then further verification through a reporter assay provided direct evidence for the specific binding. The subsequent experiments revealed that miR-455 could suppress the proliferation and migration of gastric cancer cells by negatively regulating the expression of EGFR. Furthermore, the cancer-promoting function of EGFR was further confirmed in gastric cancer cells through the silencing and overexpression of EGFR. It was demonstrated in our study that miR-455 was significantly downregulated in gastric cancer tissues and exhibited a cancer-suppressive function in gastric cancer.

EGFR is a transmembrane protein with cytoplasmic kinase activity. Homodimerization and/or heterodimerization induced by ligands causes autophosphorylation of the cytoplasmic domain of the receptor, thus activating downstream cell signaling pathways, such as the RAS-RAF-MEK-MAPK pathway $(25,26)$, the PI3K-PTEN-AKT pathway $(27,28)$ and the STAT pathway $(29,30)$, and finally facilitating the proliferation, invasion and metastasis and the suppression of apoptosis of cancer cells. Thence, suppression of EGFR is a notable method for the treatment of cancers.

There are two types of anti-EGFR targeting therapeutic agents based on the aforementioned theory: monoclonal antibodies that bind to extracellular domain of EGFR thereby preventing EGFR from binding with its endogenous ligands and tyrosine kinase inhibitors that target the cytoplasmic TK domain (31). An EXPAND study revealed that, in contrast to the favorable results in colorectal, head and neck and lung cancer, cetuxiumab, a type of monoclonal antibody, combined with chemotherapy brought no survival benefit to gastric cancer patients (32). Another monoclonal antibody, panitumumab induced a decrease in the survival of patients with gastric cancer, according to a REAL-3 study (33). Moreover, some phase II trials with EGFR TKIs, such as gefitinib or erlotinib revealed only modest benefits when used as monotherapy or combined with chemotherapy (34-36). Thus, more exploration is warranted in the targeted therapy against EGFR in gastric cancer, and negative regulation of miRNAs on target genes provide a new approach for targeted therapy. The current study demonstrated the cancer-promoting function of EGFR in gastric cancer cells and a post-transcriptional regulation strategy for EGFR mediated by miR-455, which may become a new targeted therapy for anti-EGFR therapy.

To conclude, we demonstrated that miR-455 as a tumor suppressor inhibits cell proliferation and migration in gastric cancer. In addition, our study offers a potential targeted therapeutic method against EGFR in gastric cancer mediated with the use of miR-455, which effectively inhibits the gene expression of EGFR via regulation of its $3^{\prime}$ UTR mRNA Future studies should be focused on the exploration of agents that can deliver anticancer miRNAs in vivo to suppress the expression of oncogenes.

\section{Acknowledgements}

This study was funded by grants from the National Natural Science Foundation of China (nos. 81372394, 81602158, 81572321 and 81602156) and Tianjin Health and Family Planning Commission Foundation of Science and Technology (15KG142). This study was also funded by Tianjin Science Foundation (no. 15JCYBJC28200) and Doctoral Foundation of Tianjin Medical University Cancer Institute and Hospital (B1502).

\section{References}

1. Ferlay J, Soerjomataram I, Dikshit R, Eser S, Mathers C, Rebelo M, Parkin DM, Forman D and Bray F: Cancer incidence and mortality worldwide: Sources, methods and major patterns in GLOBOCAN 2012. Int J Cancer 136: E359-E386, 2015.

2. Hashim D, Boffetta P, La Vecchia C, Rota M4, Bertuccio P, Malvezzi $M$ and Negri E: The global decrease in cancer mortality: Trends and disparities. Ann Oncol 27: 926-933, 2016.

3. Yarden $Y$ and Sliwkowski MX: Untangling the ErbB signalling network. Nat Rev Mol Cell Biol 2: 127-137, 2001.

4. Zahonero C, Sepúlveda JM and Sánchez-Gómez P: Epidermic growth factor receptor (EGFR) in glioblastomas: the mechanism of tumorigenesis and its role as a therapeutic target. Rev Neurol 61: 85-93, 2015 (In Spanish).

5. Weber KL, Doucet M, Price JE, Baker C, Kim SJ and Fidler IJ: Blockade of epidermal growth factor receptor signaling leads to inhibition of renal cell carcinoma growth in the bone of nude mice. Cancer Res 63: 2940-2947, 2003.

6. Mendelsohn J: The epidermal growth factor receptor as a target for cancer therapy. Endocr Relat Cancer 8: 3-9, 2001.

7. Wu Y, Liu H, Shi X and Song Y: Can EGFR mutations in plasma or serum be predictive markers of non-small-cell lung cancer? A meta-analysis. Lung Cancer 88: 246-253, 2015.

8. Lee HJ, Seo AN, Kim EJ, Jang MH, Kim YJ, Kim JH, Kim SW, Ryu HS, Park IA, Im SA, et al: Prognostic and predictive values of EGFR overexpression and EGFR copy number alteration in HER2-positive breast cancer. Br J Cancer 112: 103-111, 2015.

9. Nagatsuma AK, Aizawa M, Kuwata T, Doi T, Ohtsu A, Fujii H and Ochiai A: Expression profiles of HER2, EGFR, MET and FGFR2 in a large cohort of patients with gastric adenocarcinoma. Gastric Cancer 18: 227-238, 2015.

10. Weber DC, Tille JC, Combescure C, Egger JF, Laouiti M, Hammad K, Granger P, Rubbia-Brandt L and Miralbell R: The prognostic value of expression of HIF1 $\alpha$, EGFR and VEGF-A, in localized prostate cancer for intermediate- and high-risk patients treated with radiation therapy with or without androgen deprivation therapy. Radiat Oncol 7: 66, 2012.

11. Numico G, Russi EG, Colantonio I, Lantermo RA, Silvestris N, Vitiello R, Comino A, Abrate M, Zavattero C, Melano A, et al: EGFR status and prognosis of patients with locally advanced head and neck cancer treated with chemoradiotherapy. Anticancer Res 30: 671-676, 2010. 
12. Normanno N, Maiello MR and De Luca A: Epidermal growth factor receptor tyrosine kinase inhibitors (EGFR-TKIs): Simple drugs with a complex mechanism of action? J Cell Physiol 194: 13-19, 2003.

13. Ayyappan S, Prabhakar D and Sharma N: Epidermal growth factor receptor (EGFR)-targeted therapies in esophagogastric cancer. Anticancer Res 33: 4139-4155, 2013.

14. Jiang C, Chen X, Alattar M, Wei J and Liu H: MicroRNAs in tumorigenesis, metastasis, diagnosis and prognosis of gastric cancer. Cancer Gene Ther 22: 291-301, 2015.

15. Wang QX, Zhu YQ, Zhang $\mathrm{H}$ and Xiao J: Altered miRNA expression in gastric cancer: A systematic review and meta-analysis. Cell Physiol Biochem 35: 933-944, 2015.

16. Zhang XT, Zhang Z, Xin YN, Ma XZ and Xuan SY: Impairment of growth of gastric carcinoma by miR-133-mediated Her-2 inhibition. Tumour Biol 36: 8925-8930, 2015.

17. Xie J, Chen M, Zhou J, Mo MS, Zhu LH, Liu YP, Gui QJ, Zhang L and Li GQ: miR-7 inhibits the invasion and metastasis of gastric cancer cells by suppressing epidermal growth factor receptor expression. Oncol Rep 31: 1715-1722, 2014.

18. Han C, Zhou Y, An Q, Li F, Li D, Zhang X, Yu Z, Zheng L, Duan Z and Kan Q: MicroRNA-1 (miR-1) inhibits gastric cancer cell proliferation and migration by targeting MET. Tumour Biol 36: 6715-6723, 2015

19. Zheng Z, Yan D, Chen X, Huang H, Chen K, Li G, Zhou L, Zheng D, Tu L and Dong XD: MicroRNA-206: Effective inhibition of gastric cancer progression through the c-Met pathway. PLoS One 10: e0128751, 2015.

20. Peng Y, Guo JJ, Liu YM and Wu XL: MicroRNA-34A inhibits the growth, invasion and metastasis of gastric cancer by targeting PDGFR and MET expression. Biosci Rep 34: pii: e00112, 2014.

21. Liu J, Xue H, Zhang J, Suo T, Xiang Y, Zhang W, Ma J, Cai D and Gu X: MicroRNA-144 inhibits the metastasis of gastric cancer by targeting MET expression. J Exp Clin Cancer Res 34: 35, 2015.

22. Tsai MM, Wang CS, Tsai CY, Chen CY, Chi HC, Tseng YH, Chung PJ, Lin YH, Chung IH, Chen CY, et al MicroRNA-196a/-196b promote cell metastasis via negative regulation of radixin in human gastric cancer. Cancer Lett 351: 222-231, 2014.

23. An Y, Zhang Z, Shang Y, Jiang X, Dong J, Yu P, Nie Y and Zhao Q: miR-23b-3p regulates the chemoresistance of gastric cancer cells by targeting ATG12 and HMGB2. Cell Death Dis 6 : e1766, 2015

24. Zhou L, Qiu T, Xu J, Wang T, Wang J, Zhou X, Huang Z, Zhu W, Shu Y and Liu P: miR-135a/b modulate cisplatin resistance of human lung cancer cell line by targeting MCL1. Pathol Oncol Res 19: 677-683, 2013.

25. Giehl K, Skripczynski B, Mansard A, Menke A and Gierschik P. Growth factor-dependent activation of the Ras-Raf-MEK-MAPK pathway in the human pancreatic carcinoma cell line PANC-1 carrying activated K-ras: Implications for cell proliferation and cell migration. Oncogene 19: 2930-2942, 2000.
26. Troiani T, Napolitano S, Vitagliano D, Morgillo F, Capasso A, Sforza V, Nappi A, Ciardiello D, Ciardiello F and Martinelli E: Primary and acquired resistance of colorectal cancer cells to anti-EGFR antibodies converge on MEK/ERK pathway activation and can be overcome by combined MEK/EGFR inhibition. Clin Cancer Res 20: 3775-3786, 2014.

27. Davis NM, Sokolosky M, Stadelman K, Abrams SL, Libra M, Candido S, Nicoletti F, Polesel J, Maestro R, D'Assoro A, et al: Deregulation of the EGFR/PI3K/PTEN/Akt/mTORC1 pathway in breast cancer: Possibilities for therapeutic intervention. Oncotarget 5: 4603-4650, 2014.

28. Lim HJ, Crowe P and Yang JL: Current clinical regulation of $\mathrm{PI} 3 \mathrm{~K} / \mathrm{PTEN} / \mathrm{Akt} / \mathrm{mTOR}$ signalling in treatment of human cancer. J Cancer Res Clin Oncol 141: 671-689, 2015.

29. Huang L and Fu L: Mechanisms of resistance to EGFR tyrosine kinase inhibitors. Acta Pharm Sin B 5: 390-401, 2015.

30. Xu N, Wang SQ, Tan D, Gao Y, Lin G and Xi R: EGFR, Wingless and JAK/STAT signaling cooperatively maintain Drosophila intestinal stem cells. Dev Biol 354: 31-43, 2011.

31. Janmaat ML and Giaccone G: The epidermal growth factor receptor pathway and its inhibition as anticancer therapy. Drugs Today (Barc) 39 (Suppl C): 61-80, 2003.

32. Lordick F, Kang YK, Chung HC, Salman P, Oh SC, Bodoky G, Kurteva G, Volovat C, Moiseyenko VM, Gorbunova V, et al; Arbeitsgemeinschaft Internistische Onkologie and EXPAND Investigators: Capecitabine and cisplatin with or without cetuximab for patients with previously untreated advanced gastric cancer (EXPAND): A randomised, open-label phase 3 trial. Lancet Oncol 14: 490-499, 2013.

33. Waddell T, Chau I, Cunningham D, Gonzalez D, Okines AF, Okines C, Wotherspoon A, Saffery C, Middleton G, Wadsley J, et al: Epirubicin, oxaliplatin, and capecitabine with or without panitumumab for patients with previously untreated advanced oesophagogastric cancer (REAL3): A randomised, open-label phase 3 trial. Lancet Oncol 14: 481-489, 2013.

34. Wang WP, Wang KN, Gao Q and Chen LQ: Lack of EGFR mutations benefiting gefitinib treatment in adenocarcinoma of esophagogastric junction. World J Surg Oncol 10: 14, 2012.

35. Dragovich T, McCoy S, Fenoglio-Preiser CM, Wang J, Benedetti JK, Baker AF, Hackett CB, Urba SG, Zaner KS, Blanke CD, et al: Phase II trial of erlotinib in gastroesophageal junction and gastric adenocarcinomas: SWOG 0127. J Clin Oncol 24: 4922-4927, 2006.

36. Cappetta A, Lonardi S, Pastorelli D, Bergamo F, Lombardi G and Zagonel V: Advanced gastric cancer (GC) and cancer of the gastro-oesophageal junction (GEJ): Focus on targeted therapies. Crit Rev Oncol Hematol 81: 38-48, 2012. 